\title{
Analysis of the Split Ring Resonator (SRR) Antenna Applied to Passive UHF-RFID Tag Design
}

\author{
Simone Zuffanelli, Gerard Zamora, Member, IEEE, Pau Aguilà, Ferran Paredes, Ferran Martín, \\ Fellow, IEEE, Jordi Bonache, Member, IEEE
}

\begin{abstract}
An electrically small planar passive UHF-RFID tag based on an edge-coupled split ring resonator (EC-SRR) antenna is presented in this work. In order to explore the potentiality and limitations of the SRR antenna and to aid the tag design, an analytical study of the SRR radiation properties at its fundamental resonance is presented for the first time. Radiation resistance, efficiency, polarization, bandwidth and impedance matching with the RFID ASIC are treated in the study. Based on such analysis, the tag design process is presented, and a tag prototype of size $30 \mathrm{~mm} \times 30 \mathrm{~mm}\left(\lambda_{0} / 11 \times \lambda_{0} / 11\right)$ is designed to operate in the North-American UHF-RFID band (902-928 MHz) and manufactured. The measured read range is in good agreement with the simulation, and reaches $9.3 \mathrm{~m}$ at $911 \mathrm{MHz}$. The tag also features a mitigation of the blind spots, providing a minimum measured read range of $4.2 \mathrm{~m}$.
\end{abstract}

Index Terms - Split ring resonators (SRRs), electrically small antennas (ESAs), radio frequency identification (RFID).

\section{INTRODUCTION}

$\mathrm{R}$ ADIO frequency identification (RFID) is a widespread technology that allows for tagging and identification of objects by using electromagnetic waves. In the last years, the use of such technology has experienced a rapid increase, whereas the cost of the tags has dropped down, and further penetration into the market is expected for the next years [1],[2]. Typical applications of this technology are smart inventory and item tracking, among others. Passive tags operating at the UHF-RFID frequency bands $(860-960 \mathrm{MHz}$ [3]) are especially employed for this kind of applications due to the significant achievable read ranges, low cost, small dimensions, and because such tags do not need batteries. A passive UHF-RFID tag consists of an antenna matched to an application specific integrated circuit (ASIC), which contains the information about the tagged item. A passive tag is capable of using the electromagnetic energy from the reader to activate the chip, which generates a modulated backscattered signal to the reader. Typical peak read ranges of UHF-RFID tags are in the order of $6^{-12} \mathrm{~m}$, depending on the country regulations (i.e.

Manuscript received February 16, 2015. This work has been supported by MINECO-Spain (project TEC2013-40600-R COM-SEN-RFID), by FEDER funds, and by Generalitat de Catalunya (project 2014SGR-157). Ferran Martín is in debt to ICREA for supporting his work and by awarding him with an ICREA Academia Award.

The authors are with GEMMA/CIMITEC, Departament d'Enginyeria Electrònica, Universitat Autònoma de Barcelona, 08193 Bellaterra, Spain (email: Jordi.Bonache@uab.es). maximum allowed equivalent isotropic radiated power -EIRP - value), tag characteristics (i.e. antenna performance and chip sensitivity) and orientation, item material, and environmental conditions [4].

One of the most challenging aspects regarding passive UHFRFID tag design is size reduction. This feature is required as far as the dimensions of the tagged object are small. Note that tag area is limited by such dimensions, which may be much smaller than the wavelength at the working frequency. Since the RFID ASIC is always far smaller than the antenna, the tag dimensions are determined by the size of the antenna and the matching network (if it is present). Several techniques have been described in the literature in order to reduce antenna dimensions, such as meandering and inverted-F configurations [5]. Recently, metamaterial-inspired resonators have also been used to shrink tag dimensions [6], due to their very small dimensions in terms of wavelength. Among these, the edge coupled split ring resonator (EC-SRR), first proposed by Pendry [7], has been also reported as a standalone radiating particle for UHF-RFID tags [8],[9]. However, in [8] the authors declare that the radiating part is only the external ring, being the internal ring much smaller and dedicated to the impedance matching functionality between the ASIC and the antenna. Due to the significant difference between the internal and external radii, the SRR does not behave as its quasi-static analysis predicts [10], i.e., the SRR electrical size in [8] is not reduced as compared to that of a single ring resonator (where $r_{0} \approx \lambda_{0} /(4 \pi), r_{0}$ and $\lambda_{0}$ being the ring radius and the freespace wavelength, respectively). Moreover, many important aspects relative to tag performance, e.g., radiation efficiency, bandwidth and read range are not treated in the works mentioned above. For these reasons, further study is needed in order to understand the capacities and limitations of SRR antennas applied to the design of electrically small passive UHF-RFID tags. Additionally, the analysis of the radiation properties of the SRR at its fundamental resonance is of interest within the context of electrically small antennas (ESAs), and hence this work goes beyond the mere application of such analysis to the design of compact UHF-RFID tags.

The work is organized as follows. An analytical study of the radiation properties of the SRR antenna is carried out in Section II, which is also dedicated to the impedance matching strategy between the RFID ASIC and the antenna, and to the analysis of the maximum achievable bandwidth for a given tag size. Based on the presented analysis, a tag prototype is designed with the aid of EM simulation software, and the simulation results are presented in Section III. In order to 
validate the results, the prototype is fabricated and the antenna input impedance, along with the radiation pattern and the tag read range, is measured and compared to simulated results in Section IV. Finally, the main conclusions are highlighted in Section V.

\section{ANALYTICAL STUDY}

\section{A. Radiation resistance and far-field pattern}

The topology of the EC-SRR is depicted in Fig. 1. The electric current and charge density distribution at each ring at the fundamental (first) resonance can be found from the quasistatic analysis presented in [10], which takes advantage of the fact that the particle is electrically small. From such analysis, it was found that the sum of the currents in the outer and inner rings (flowing in the same direction) is constant along the whole circumference (i.e., the total current, $i_{0}$, in any cross section of the SRR does not depend on the position angle $\varphi$ ). This means that the structure can be seen as an electrically small constant current loop, providing an axial magnetic dipole moment given by

$$
\boldsymbol{m}=\pi r_{0}^{2} i_{0} \cos \left(\omega_{0} t\right) \hat{\mathbf{z}}
$$

where $r_{0}$ is the arithmetic mean of the internal and external radii and $\omega_{0}$ is the working angular frequency. The power radiated by such a magnetic moment of amplitude $m_{0}$ can be written as [11]

$$
P_{\text {rad }}^{\mathrm{M}}=\frac{Z_{0} k^{4} m_{0}^{2}}{12 \pi},
$$

where $Z_{0}$ is the free-space impedance and $k$ is the free-space wavenumber. Therefore, the radiation resistance associated to $\boldsymbol{m}$, referred to the current amplitude $i_{0}$, can be found to be:

$$
R_{\mathrm{rad}}^{\mathrm{M}}=\frac{8}{3} \pi^{5} Z_{0}\left(\frac{r_{0}}{\lambda_{0}}\right)^{4} \text {. }
$$

Since the size of the SRR mean radius in terms of wavelength is in the order of $1 / 20$, the radiation resistance associated to the axial magnetic dipolar moment $\boldsymbol{m}$ is in the order of $1-2 \Omega$, though it strongly depends on the particle size (i.e., the substrate thickness and permittivity).

Let us now evaluate the radiation resistance associated to the electric dipole moment tangential to the plane of the particle, which arises from the bi-anisotropy of the EC-SRR. The quasistatic analysis assumes that the current at each ring depends linearly on $\varphi$ [10]. Therefore, the current in the external ring (A) can be written as

$$
\boldsymbol{i}_{A}(\varphi, t)=i_{0}\left(1-\frac{|\varphi|}{\pi}\right) \cos \left(\omega_{0} t\right) \hat{\boldsymbol{\varphi}}
$$

where $\varphi \in[-\pi, \pi]$. By applying the charge conservation law, the linear charge density on the external ring can be written as:

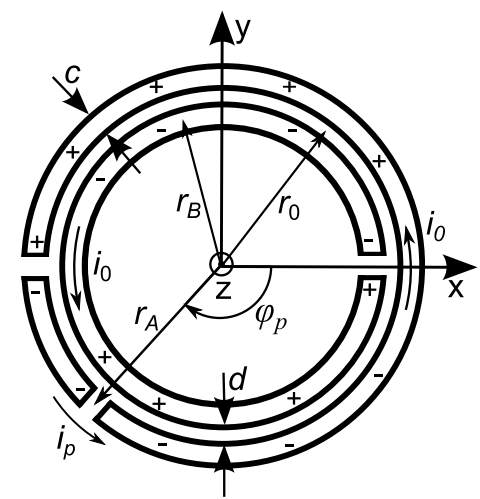

Fig. 1. Topology and electric current and charge distribution of the EC-SRR at its fundamental resonance (note that for the analysis in this section, the slit in the external ring, positioned at $\varphi_{p}$, is not present, thus obtaining the typical topology of an EC-SRR. However, such a slit is used to feed the antenna, as discussed later).

$$
\lambda_{A}(\varphi, t)=\frac{i_{0}}{\pi r_{A} \omega_{0}} \sin \left(\omega_{0} t\right) \operatorname{sgn}(\varphi) .
$$

Since expression (5) is odd with respect to the position angle $\varphi$, the total electric dipole moment associated to ring $\mathrm{A}$ is the sum of the infinitesimal dipole moments directed along the $\hat{\boldsymbol{y}}$ direction, namely:

$$
\boldsymbol{p}_{A}=\int_{0}^{\pi} \lambda_{A}(\varphi, t) 2 r_{A}^{2} \sin \varphi d \varphi \hat{\boldsymbol{y}} .
$$

By integrating expression (6) we obtain

$$
\boldsymbol{p}_{A}=\frac{4}{\pi} \frac{r_{A}}{\omega_{0}} i_{0} \sin \left(\omega_{0} t\right) \hat{\boldsymbol{y}} .
$$

The internal ring $\mathrm{B}$ (with radius $r_{B}$ ) exhibits an electric dipole moment with the same orientation, but presents opposite sign, due to the 180 degrees shift in the ring cut position. Therefore, the total electric dipole moment is obtained by subtracting their amplitudes. By assuming the hypothesis of strong coupling between rings, which is normally satisfied at the first resonance, the current in the internal ring has roughly the same amplitude $i_{0}$ as the current in the outer ring. Taking into account that $r_{A}-r_{B}=c+d$, the total electric dipole moment is

$$
\boldsymbol{p}=\frac{4}{\pi \omega_{0}}(c+d) i_{0} \sin \left(\omega_{0} t\right) \hat{\boldsymbol{y}} .
$$

Then, by using the Larmor equation [12] for the radiated power by a small electric dipole moment of amplitude $p_{0}$, that is

$$
P_{\text {rad }}^{\mathrm{E}}=c_{0}^{2} \frac{Z_{0} k^{4} p_{0}^{2}}{12 \pi},
$$

where $c_{0}$ is the speed of light in vacuum, the radiation resistance associated to $\boldsymbol{p}$, and referred to the maximum current $i_{0}$ can be expressed as follows:

$$
R_{\mathrm{rad}}^{\mathrm{E}}=\frac{32}{3 \pi} Z_{0}\left(\frac{c+d}{\lambda_{0}}\right)^{2} .
$$

Let us now compare (10) with (3). Since the typical values of $c+d$ in terms of wavelength are in the order of $1 / 100$, that is, five or more times smaller than the mean radius $r_{0}$, the 
radiation resistance associated to the electric dipole moment is in the order of $0.1 \Omega$, which is an order of magnitude smaller than the radiation resistance related to the magnetic dipole moment of the SRR. Therefore, the radiation resistance of the particle can be approximated by the resistance associated to the axial magnetic moment, that is:

$$
R_{\mathrm{rad}} \approx R_{\mathrm{rad}}^{\mathrm{M}},
$$

which produces a linearly polarized radiation with a maximum in the $x y$-plane and a theoretical directivity of $1.5(1.75 \mathrm{dBi})$, as in the case of an electrically small current loop (more precisely, the presence of the electric dipole moment sets the maximum radiation in the direction of the $x$-axis, as it will be detailed below). Nevertheless, the relation between the electric and magnetic radiation is useful to evaluate the read range of the tag in the axial direction. In fact, instead of presenting a null read range for $\theta=0^{\circ}$ (where $\theta$ is the polar angle measured from the $z$-axis), where the magnetic radiation vanishes, the tag is expected to show a residual read range provided by the electric radiation. The ratio between the resistances (10) and (3), that is

$$
X P O L_{\max }=\frac{R_{\mathrm{rad}}^{E}}{R_{\mathrm{rad}}^{M}}=\frac{4}{\pi^{6}}\left(\frac{c+d}{\lambda_{0}}\right)^{2}\left(\frac{r_{0}}{\lambda_{0}}\right)^{-4},
$$

is useful to quantify the read range in the direction of the minimum radiation. In fact, by assuming that the radiation pattern of the magnetic and electric moments present the same shape (i.e. both are elemental radiators), the gain in the axial direction is found to be $X P O L_{\max } /\left(1+X P O L_{\max }\right)$ times the gain in the main direction ( $x$-axis), where both the electric and magnetic dipoles contribute to radiation. The read range, as a function of the tag antenna gain, can be written by using the well-known expression [4]

$$
R R=\frac{\lambda_{0}}{4 \pi} \sqrt{\frac{E I R P \cdot G_{r} \cdot \tau}{P_{\mathrm{th}}}},
$$

where EIRP is the equivalent isotropic radiated power, $\lambda_{0}$ is the free-space wavelength at the working frequency, $G_{r}$ is the gain of the tag antenna, $\tau=\left(1-\left|s_{11}\right|^{2}\right)$ is the power transmission coefficient, and $P_{\text {th }}$ is the ASIC read sensitivity. As can be seen in (13), the read range depends on the square root of the antenna gain. Therefore, the read range $R R_{z}$ in the axial direction can be referred to the maximum read range $R R_{\mathrm{x}}$ as:

$$
R R_{z}=R R_{\mathrm{x}} \sqrt{\frac{X P O L_{\max }}{1+X P O L_{\max }}}
$$

which also corresponds to the minimum read range of the tag. Its quantification is interesting because in most RFID applications the tags are required to respond independently of their orientation, generally unknown a priori, and therefore the presence of blind spots should be minimized.

The value of $X P O L_{\max }$ is also useful to predict the polarization of the radiated fields. In fact, if the radiation produced by the magnetic dipole moment is defined as copolar (being the main radiation mechanism), the contribution from the electric dipole moment is entirely cross-polar in the $y z$-plane, due to the orthogonality and the $90^{\circ}$ phase shift (see Eq. 1 and 8) between the two radiators. Therefore, the maximum value of the cross-polar component in the whole radiation diagram, normalized to the maximum value of the co-polar, is equal to $X P O L_{\max }$. Also, in the $y z$-plane, four zones where the lineal axial ratio decays down to unity exist. In fact, since the co-polar radiation is proportional to $\sin ^{2}(\theta)$ and the cross-polar is proportional to $\cos ^{2}(\theta)$, a circular polarization is generated at the four angles $\theta_{c}=\left\{ \pm t g^{-1}\left(\sqrt{X P O L_{\max }}\right), \pm t g^{-1}\left(\sqrt{X P O L_{\max }}\right)+\pi\right\}$, and the axial ratio maintains under an arbitrary level $A R_{0}$ (e.g., $A R_{0}=10 \mathrm{~dB}$ can be considered as the boundary between elliptical and linear polarization) in each of the four regions around $\theta_{c}$ and subtending an angle equal to $\Delta \theta_{\text {ell }}=\operatorname{tg}^{-1}\left(A R_{0} \sqrt{X P O L_{\max }}\right)-t g^{-1}\left(A R_{0}^{-1} \sqrt{X P O L_{\max }}\right)$. It has been verified by simulation that, for values of $A R_{0}$ in the order of $10 \mathrm{~dB}$, each of these regions describes approximately a cone in the radiating sphere, thus enclosing a solid angle $\Omega_{\mathrm{ell}} \approx 2 \pi\left[1-\cos \left(\Delta \theta_{\mathrm{ell}} / 2\right)\right]$. For $X P O L_{\max }<<1$, which is satisfied according to (12), the solid angle $\Omega_{\text {ell }}$ is much smaller than $4 \pi$, and therefore the tag radiation can be considered linear on most of the radiating sphere, as it is generally required for an RFID tag in order to maximize the read range. On the other hand, very small values of $X P O L_{\max }$ provide a low residual read range in the axial direction, as expressed in (14), suggesting that a tradeoff between these two factors has to be considered at the design stage.

\section{B. Radiation efficiency}

In this section we will analyze the effect of the ohmic losses introduced by conductors on the tag radiation efficiency. As referred in [13], the loss resistance of the SRR can be approximated to the one of a constant current loop with length $l=2 \pi r_{0}$ and width $c$. When the metal thickness is larger than the skin depth $\delta$ at the working frequency, which is in the order of $2 \mu \mathrm{m}$ for annealed copper at the UHF-RFID band, the loss resistance can be expressed as [13]

$$
R_{\mathrm{loss}}=\frac{\pi r_{0}}{c \delta \sigma},
$$

where $\sigma$ is the conductivity of the metal. A shunt LC circuit model is often reported in order to describe the impedance behavior of the SRR around its first resonance [10]. However, when the excitation port is placed across a cut opened in one of the rings (Fig. 1), and losses are taken into account, a series RLC circuit is the adequate model to describe the particle. In this case, the loss resistance is series connected to the radiation resistance, and therefore the radiation efficiency can be evaluated as $\eta_{\text {rad }}=\left[1+R_{\text {loss }} / R_{\text {rad }}\right]^{-1}$, obtaining

$$
\eta_{\mathrm{rad}}=\left[1+\frac{3}{8} \frac{c_{0}^{4} \sqrt{\pi \mu_{0}}}{\pi^{4} Z_{0}}\left(c r_{0}^{3} \sqrt{f_{0}^{7} \sigma}\right)^{-1}\right]^{-1},
$$

where $\mu_{0}$ is the permeability of free-space. Expression (16) can be rewritten in a simpler form, by fixing the metal conductivity to that of copper $\left(5.96 \cdot 10^{7} \mathrm{~S} / \mathrm{m}\right)$, and the frequency to that of 
the central North-American UHF-RFID band ( $\left.f_{0}=915 \mathrm{MHz}\right)$, obtaining

$$
\eta_{\text {rad }}=\left[1+\frac{920}{c r_{0}^{3}}\right]^{-1},
$$

where all the dimensions are expressed in mm. Expression (17) is very useful to the design of an efficient tag based on this kind of antenna. Firstly, it suggests that the radiation efficiency is strongly dependent on particle size. For this reason, the substrate should be chosen to be thin and with a low relative permittivity. Moreover, expression (17) shows that the radiation efficiency directly depends on the strip width, and sufficiently high efficiency can be obtained with reasonable values of $c$. For a $r_{0}$ value of $15 \mathrm{~mm}$ (which corresponds to approximately $1 / 20$ wavelength at $f_{0}=915 \mathrm{MHz}$ ), a $2.2 \mathrm{~mm}$ width provides $\eta_{\text {rad }}=89 \%$, and consequently a gain of $G_{r}=1.25 \mathrm{~dB}$ (considering a directivity of 1.5), which is promising for an UHF-RFID tag of this size.

It is worth to mention that the previous analysis does not take into account dielectric losses, which will slightly decrease the overall efficiency as a function of the substrate thickness and loss tangent.

\section{C.Impedance matching and bandwidth}

Let us discuss the impedance matching between the RFID ASIC and the SRR antenna, which is a fundamental aspect for the final tag performance (see Eq. 13). The input impedance of an RFID ASIC is typically capacitive, with a reactive part $X_{C}$ one order of magnitude greater than the resistive part $R_{C}$, which is in the order of 15-30 $\Omega$. Now, since the SRR presents a narrow-bandwidth (as it will be detailed later) series resonance, it is expected that nearly beyond resonance the inductive reactance will match the capacitive reactance of the chip. At that frequency, the reflection coefficient notch depth (and thus the value of $\tau$ ) depends on the ratio between the input resistance $R_{\text {in }}$ and the chip resistance $R_{C}$. If the port is placed in the external ring at $\varphi=0^{\circ}$, where a current maximum $i_{0}$ occurs, the input resistance is equal to $R_{0}=\left(R_{\mathrm{rad}}+R_{\text {loss }}\right)$, which is in general much lower than $R_{C}$, resulting in a significant impedance mismatch. However, it is possible to change the port position in order to increase the input resistance $R_{\text {in }}(\varphi)$ until reaching a good impedance matching without the need of an external matching network. In fact, as reported in [14], the following expression provides the input resistance, as a function of the port position, for asymmetrically fed wire antennas:

$$
R_{\mathrm{in}}(\varphi)=\frac{i_{0}^{2}}{i_{A}^{2}(\varphi)} R_{0} .
$$

By substituting expression (4) for the current profile $i_{A}$ in (18), the port angle $\varphi=\varphi_{p}$ providing $R_{\text {in }}=R_{C}$ is found to be

$$
\varphi_{p}= \pm \pi(1-\sqrt{\alpha}),
$$

where $\alpha$ is a scaling factor, and is defined as $\alpha=R_{0} / R_{C}$. The proposed method allows overcoming the limitations reported in [15] on the impedance matching between an RFID ASIC and an SRR antenna.
Let us now discuss the maximum bandwidth that can be obtained with conjugate impedance matching. The physical limitations affecting linear-polarized planar electrically small antennas were first studied by Gustafsson [16], as an extension of the work of Wheeler [17] and Chu [18]. It was found that the minimum radiation quality factor $Q_{\text {rad }}$ for a planar antenna enclosed in a sphere of a radius $r_{\text {ext }}$ is at least $9 \pi / 8$ times higher than the lower bound for any antenna enclosed in the same sphere [16],[19], which can be approximated to $Q_{\text {rad }} \geq\left(k_{0} \cdot r_{\text {ext }}\right)^{-3}[20]$. Therefore, taking into account that $Q=Q_{\text {rad }} \cdot \eta_{\text {rad }}$, it follows that

$$
Q \geq \frac{9}{8} \pi \eta_{\text {rad }}\left(k_{0} r_{\text {ext }}\right)^{-3},
$$

which, in terms of fractional half-power bandwidth assuming conjugate matching, can be expressed as [21]

$$
F B W \approx \frac{2}{Q} \leq \frac{128}{9} \pi^{2} \eta_{\text {rad }}^{-1}\left(\frac{r_{\text {ext }}}{\lambda_{0}}\right)^{3},
$$

where $r_{\mathrm{ext}}$ is the radius of the smallest sphere enclosing the antenna and, in our case, corresponds to the external radius of the outer ring $\left(r_{\mathrm{ext}}=r_{0}+c+d / 2\right)$. Expression (21) is valid for $Q>>1$, a condition normally satisfied for an electrically small antenna with acceptable radiation efficiency. Taking into account that, as said before, the mean radius (and consequently the external radius) is in the order of $1 / 20$ times the free-space wavelength and the radiation efficiency approaches unity, the fractional bandwidth is expected to be $F B W \leq 2 \%$, corresponding to roughly $20 \mathrm{MHz}$ at the UHF-RFID band.

Equation (21) sets the lower bound for the quality factor of any small antenna confined to a planar geometry. In practice, the SRR antenna is expected to provide a higher quality factor, which can be predicted on the basis of its equivalent circuit model near the resonance, i.e. a series RLC resonator, providing $Q_{\mathrm{rad}}=\omega_{0} L / R_{\mathrm{rad}}$. The value of the EC-SRR selfinductance $L$ can be computed as the average of the inductances of both rings [10], i.e. the inductance of a loop with radius $r_{0}$ and width $c$. A commonly used approximated expression for the inductance of a circular loop is $L \approx \mu_{0} r_{0}\left[\ln \left(16 r_{0} / c\right)-2\right]$, which assumes a circular section with radius $c / 2$. Hence, by using (11) for the radiation resistance, the antenna SRR bandwidth, in the case of conjugate matching, can be approximated to

$$
F B W \approx \frac{8}{3} \pi^{4} \eta_{\mathrm{rad}}^{-1}\left[\ln \left(16 \frac{r_{0}}{c}\right)-2\right]^{-1}\left(\frac{r_{0}}{\lambda_{0}}\right)^{3} .
$$

Expression (22) assumes that the input resistance of the port does not vary in frequency. This condition is not strictly satisfied in the case of RFID ASICs, which are modeled by shunt RC circuits. However, the variation of the ASICs input resistance $R_{C}$ is usually very small within the UHF-RFID band [22], so that (22) can be used for the tag bandwidth to a good degree of approximation. For practical cases, the values found by using (22) are in the order of $F B W \approx 0.8-1 \%$ when good 


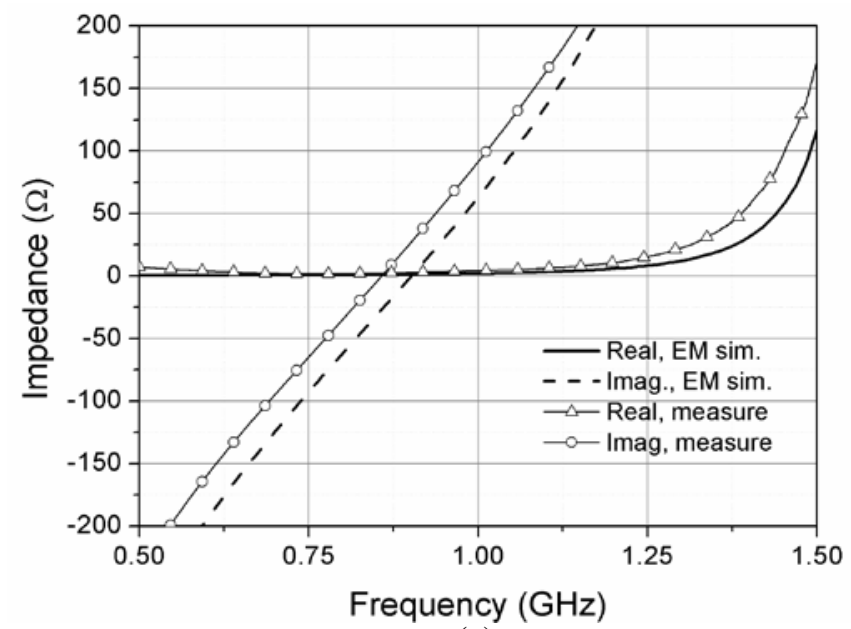

(a)

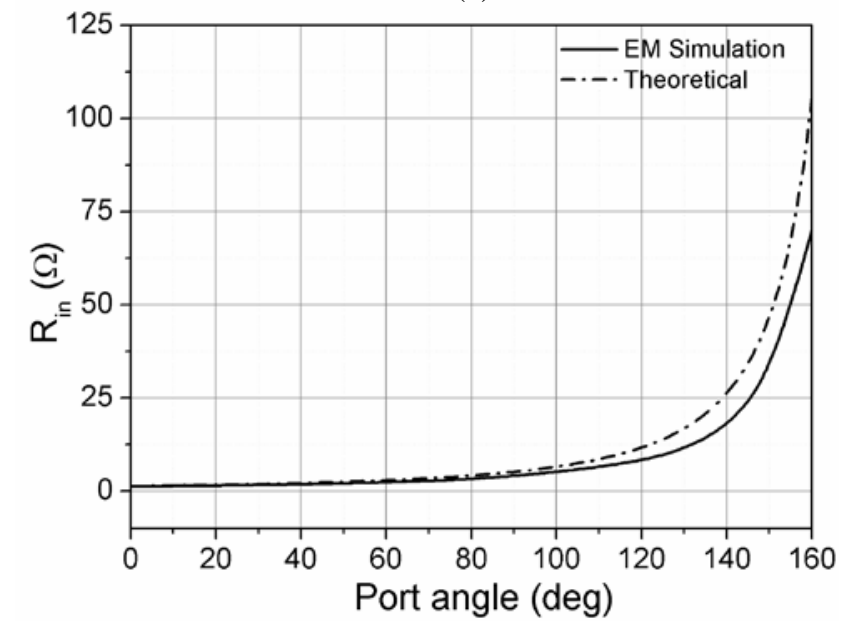

(b)

Fig. 2. (a) Input impedance of the proposed SRR antenna fed at the center of the external ring, and (b) input resistance as a function of the port position. Dimensions are $r_{0}=13.1 \mathrm{~mm}, c=2.2 \mathrm{~mm}$ and $d=0.33 \mathrm{~mm}$.

radiation efficiency is obtained. Although this value does not allow a worldwide operation of the tag, it is large enough for ensuring its correct operation in one of the UHF-RFID bands. It is worth to mention that ohmic and dielectric losses decrease the overall quality factor, thus increasing the bandwidth at the expense of a reduction of the tag read range.

\section{TAG DESIGN AND SIMULATION}

In this section the tag prototype design process, based on the analysis of the previous section and aided by means of EM simulations, is presented. The tag was designed to be used in the North-American UHF-RFID band (902-928 MHz), which is centered at $f_{0}=915 \mathrm{MHz}$. The simulations were performed by means of the commercial software Advanced Design System. The RFID ASIC used for the prototype is the Alien Higgs 3 with SOT-323 packaging, with an input impedance of $Z_{C}=R_{\mathrm{C}}+j X_{C}=25-j 190 \Omega$ at $f_{0}$, and a minimum sensitivity of $P_{\mathrm{th}}=-15 \mathrm{dBm}$.

As a first step, a substrate was chosen. As said in Section II.B, the substrate should be chosen to be thin and with a low relative permittivity. In our case, the Arlon CuClad 250LX was selected, presenting a thickness $h_{s}=0.49 \mathrm{~mm}$, a relative

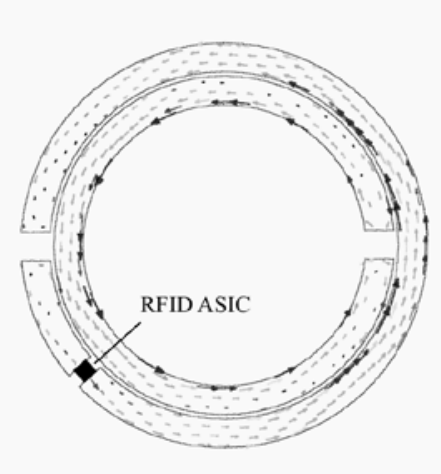

(a)

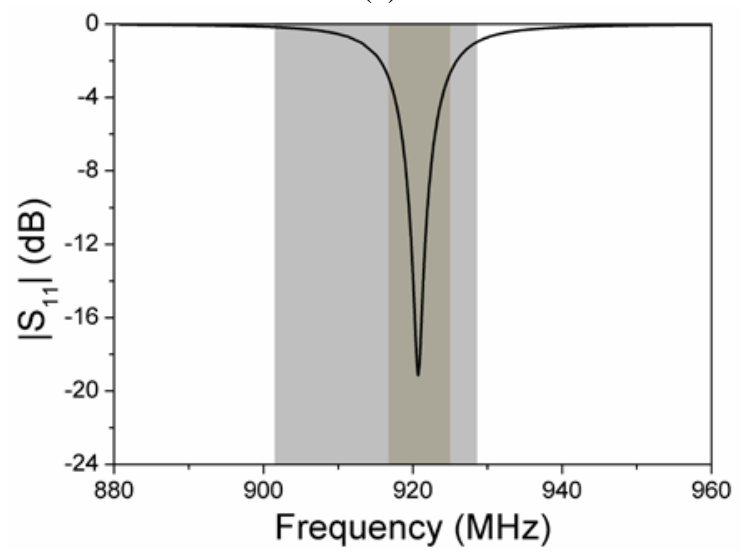

(b)

Fig. 3. Simulated (a) current distribution in the SRR tag antenna and (b) power reflection coefficient of the proposed tag. The half-power bandwidth is depicted in dark grey, the North-American UHF RFID band is depicted in light grey.

permittivity $\varepsilon_{r}=2.42$, a loss tangent $\delta_{\text {loss }}=0.0018$ and a copper layer with a thickness $h=35 \mu \mathrm{m}$. Thus, the SRR was designed as follows. The differential $50 \Omega$ port was connected through a $1 \mathrm{~mm}$ wide cut opened on the external ring at the position $\varphi_{p}=0$, so that the input resistance $R_{\text {in }}$ is equal to $R_{0}$ by definition. The ring width was set to $c=2.2 \mathrm{~mm}$, in order to obtain an acceptable radiation efficiency (see Section II.B). A combination of mean radius $r_{0}$ and distance $d$ was chosen in order to locate the first resonance just below the working frequency $f_{0}$, according to the considerations exposed in Section II.C. The set of values $r_{0}=13.1 \mathrm{~mm}\left(\lambda_{0} / 25\right)$ and $d=0.33 \mathrm{~mm}$ (consequently, the external radius value is $r_{\text {ext }}=15.46 \mathrm{~mm}$ ) provided resonance at $f_{r}=900 \mathrm{MHz}$, as suggested by the simulation results depicted in Fig. 2(a). The simulated value of the input resistance was $R_{0}=1.3 \Omega$ [Note that, by combining (11) and (15), a theoretical value, which neglects the dielectric losses, of $R_{0}=1 \Omega$ is obtained]. Therefore, the scaling factor was determined as $\alpha=R_{0} / R_{C}=1.3 / 25=0.052$ and, by using (19), the port positions providing conjugate matching were found to be $\varphi_{p}= \pm 139^{\circ}$.

A second set of simulations, which provided the value of the antenna input resistance as a function of the port position angle, was performed in order to validate (18) and (19). The results, depicted in Fig. 2(b), confirm the validity of the 
proposed impedance matching method, though the simulated values of the input resistance are slightly smaller compared to the theoretical values. Such a discrepancy should be mainly attributed to the approximation of the current profile along the ring, expressed by (4).

The last simulation was performed by placing the port cut at the position $\varphi_{p}=-139^{\circ}$, and modeling the port by the RFID ASIC equivalent circuit model, which is an RC shunt circuit, with values $R_{c}=1500 \Omega, C_{c}=0.9 \mathrm{pF}$ (as mentioned above, it provides $Z_{C}=R_{C}+j X_{C}=25-j 190 \Omega$ at $f_{0}$ ). The final tag layout, along with the simulated current distribution at the working frequency, is depicted in Fig. 3(a).

The simulation results, in terms of power reflection coefficient, are depicted in Fig. 3(b). As expected, good impedance matching [ $-19 \mathrm{~dB}$, which in RFID provides $99.4 \%$ of the read range obtained with conjugate matching (see Eq. 13)] is obtained closely above the SRR resonant frequency, at $920 \mathrm{MHz}$, which is close to the central frequency of the NorthAmerican UHF-RFID band. The half-power bandwidth of the simulated tag (depicted in grey) is $B W=8 \mathrm{MHz}$.

Let us examine the radiation pattern provided by the EM simulation of the tag [Fig. 4(a)]. The pattern was normalized to the simulated directivity level $D_{0}=1.6 \mathrm{~dB}$. As expected, the polarization and shape of the radiated fields correspond to that of a current loop laying in the $x y$-plane, i.e. the magnetic field is oriented towards $\hat{\theta}$, the electric field is oriented towards $\hat{\varphi}$, and the radiation is almost perfectly omnidirectional in the $E$ plane $\left(\theta=90^{\circ}\right)$, with a maximum along the $x$-axis due to the contribution of the electric dipole moment radiation. The radiation in the $H$-plane (defined as $\varphi=0^{\circ}$ ) is also in very good agreement with the pattern of a small current loop. The radiation efficiency was found to be $\eta_{\mathrm{rad}}=67 \%$. In comparison, by using (17) with the given SRR dimensions, an efficiency of $84 \%$ is predicted. The difference is attributed to the presence of the substrate, which, as confirmed by means of simulations with lossless substrates, introduces significant amount of dielectric losses.

The realized gain provided by simulation is $-0.1 \mathrm{~dB}$. Therefore, the maximum read range can be predicted by using (13), obtaining $R R_{\mathrm{z}}=9.2 \mathrm{~m}$. The tag read range in the axial direction can be predicted directly, i.e. by using (13) with the simulated gain in the $z$-direction $(-8.7 \mathrm{~dB})$, providing $R R_{\mathrm{z}}=3.4 \mathrm{~m}$.

The simulated value of $X P O L_{\max }$ can be directly read in the normalized pattern in the $y z$-plane [Fig. 4(b)], where the radiation from the electric and magnetic moments is decoupled, as predicted in Section II.A). Note that the simulated value $(-9 \mathrm{~dB})$ is very near to the theoretical value ( $-10 \mathrm{~dB})$, obtained from (12). A validation of expression (14), and, consequently, of the SRR antenna modeling as a combination of two elemental radiators (see Section II.A), can be done by evaluating the tag read range in the axial direction on the basis of $X P O L_{\max }$ and $R R_{x}$. The result is $R R_{z}=3.2 \mathrm{~m}$, which is very near to the value predicted above.

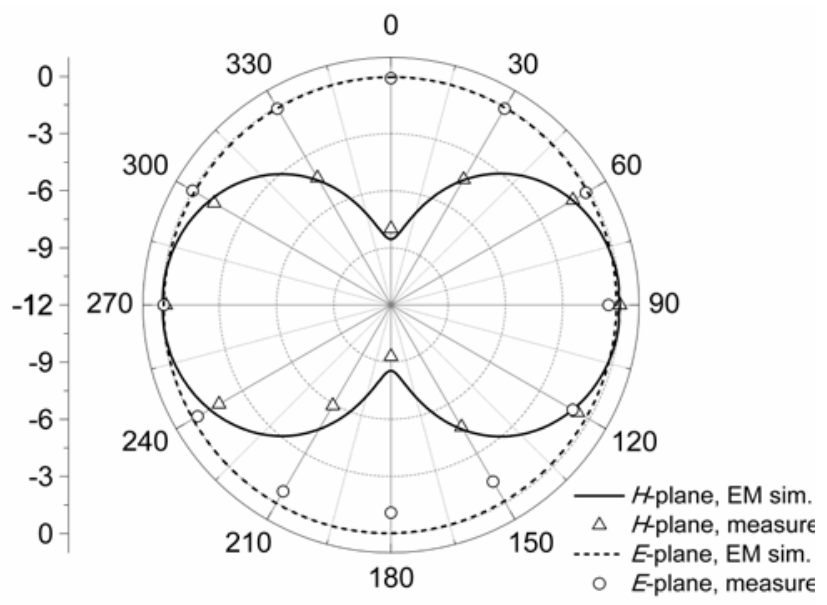

(a)

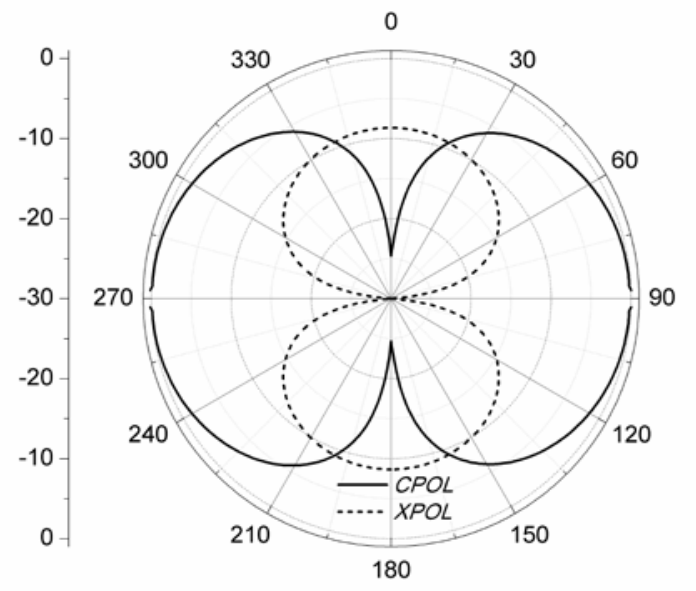

(b)

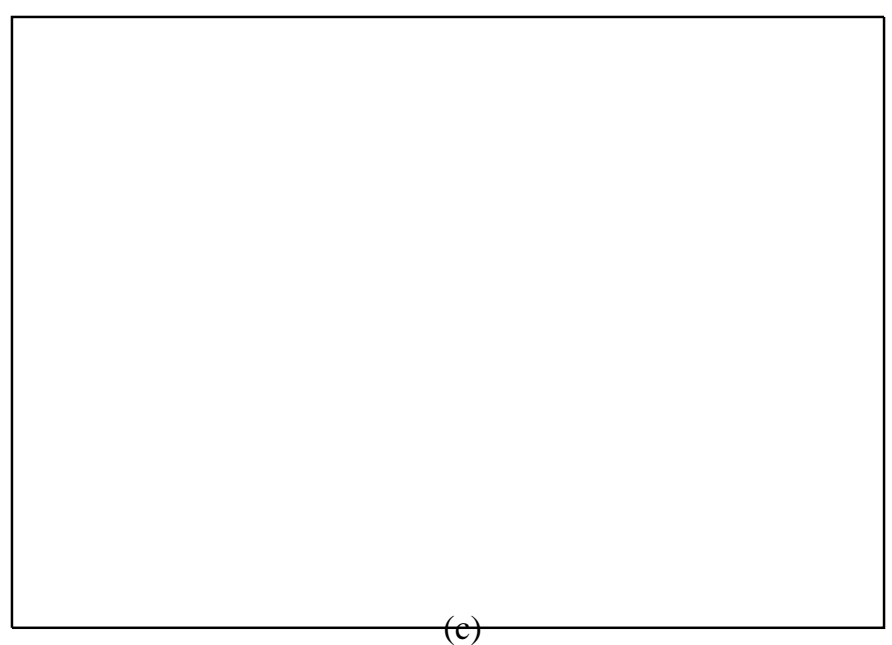

Fig. 4. (a) Normalized radiation pattern in the $H$-plane $\left(\varphi=0^{\circ}\right)$ and $E$-plane $\left(\theta=90^{\circ}\right)$, simulated (b) co-polar and cross-polar pattern in the $y z$-plane and (c) axial ratio in the $y z$-plane [low axial-ratio $(<10 \mathrm{~dB})$ region in grey].

The simulated axial ratio in the $y z$-plane is depicted in [Fig. 4(c)]. Each of the four grey regions represents the cut of the conical zones presenting axial ratio $A R_{0}$ lower than $10 \mathrm{~dB}$ (which has been considered the limit for linear polarization), and extends for $\Delta \theta_{\text {ell }}=42^{\circ}$. Therefore, each conical zone subtends a solid angle of $\Omega_{\text {ell }}=0.42 \mathrm{sr}$ in the three-dimensional 


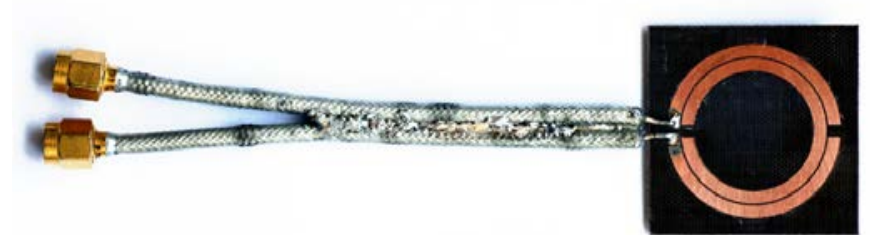

(a)

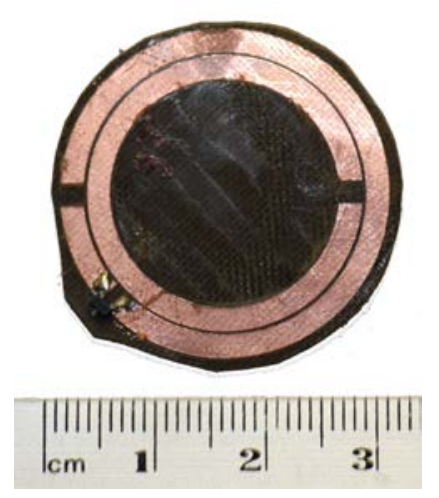

(b)

Fig. 5. (a) SRR antenna input impedance measurement assembly and (b) tag prototype ( $97 \%$ scaled).

space. As a result, the tag polarization can be considered linear on $87 \%$ of the radiation sphere.

\section{FABRICATION AND MEASUREMENT}

\section{A. Measurement setup}

The antenna impedance measurement setup consisted of an Agilent N5221A two-ports network analyzer, connected to the SRR antenna through a test fixture based on two semi-rigid coaxial cables soldered together [Fig. 5(a)]. Based on the measured scattering parameters, the differential input impedance was evaluated by using the technique described in [23].

The tag read range measurement setup [Fig. 6(a)] consisted of an Agilent N5182A vector signal generator, capable of generating RFID interrogation frames, connected to a TEM cell (Wavecontrol WaveCell) by means of a $50 \Omega$ coaxial cable. A circulator was used to send the backscattered signal from the TEM cell to an Agilent N9020A signal analyzer in order to decode the digital RFID frames generated by the tag, which was placed inside the TEM cell on top of a foam structure and oriented along the desired direction. To measure the tag read range, an RFID interrogation frame was sent to the TEM cell at different power levels in order to determine the minimum power level $P_{\min }$ required to activate the tag, that is, to receive a backscattered response frame. An electric field probe (Wavecontrol EFCube) was then placed at the tag position in order to measure the root mean square of the electric field generated by the interrogation frame at the power $P_{\min }$. Therefore, being $E_{\mathrm{rms}}$ the minimum electric field required for the tag operation, it is possible to calculate the read range directly from its value. In fact, the average power density $S$ associated to a plane wave is determined from the value of the electric field according to

$$
S=\frac{E_{\text {rms }}^{2}}{Z_{0}}
$$

For a radiating antenna it is possible to calculate the far field Poynting vector module in a given direction as

$$
S=\frac{P_{t} G_{t}}{4 \pi r^{2}}=\frac{E I R P}{4 \pi r^{2}},
$$

where $P_{t}$ is the total transmission power, $G_{t}$ is the antenna gain and $r$ is the distance from the antenna. Thus, the read range $R R$ can be obtained by equating (23) and (24), resulting in

$$
R R=\frac{\sqrt{30 E I R P}}{E_{\text {rms }}} .
$$

The method described above was then repeated for each frequency of interest (e.g., at $1 \mathrm{MHz}$ steps) in order to obtain the read range curve as a function of frequency.

The TEM cell was also used in order to measure the tag normalized radiation pattern at a given frequency. To this end, the tag orientation inside the cell was swept, with a step of $30^{\circ}$, by means of a foam rotatable support. At each angle, the value of the power $P_{\min }$ required to activate the chip was measured. The normalized pattern was then obtained dividing the required power $P_{\min , 0}$ at the maximum gain direction by the minimum required power $P_{\min , i}$ at the $i$-esim direction.

\section{B. Fabrication, measurements and discussion}

To validate the simulated results, the layout described in Section III was fabricated by means of a PCB drilling machine (LPKF-H100). In order to measure the SRR antenna input impedance, a first prototype was fabricated with the input port placed at the center of the external ring $(\varphi=0)$ [Fig. 5(a)]. The results, depicted at Fig. 2(a), exhibit good agreement with simulations. Nevertheless, the first resonance according to measurements is located at $857 \mathrm{MHz}$, i.e. $43 \mathrm{MHZ}$ below the simulated value (900 MHz).

The final tag prototype [Fig. 5(b)] was then fabricated by applying a scaling factor (0.97), in order to compensate the frequency shift detected in the measurements. The final tag dimensions are $r_{0}=12.7 \mathrm{~mm}, c=2.1 \mathrm{~mm}, d=0.32 \mathrm{~mm}$ and consequently $r_{\text {ext }}=15 \mathrm{~mm}\left(\lambda_{0} / 22\right)$. The ASIC was soldered across the cut opened at the position $\varphi_{p}=-139^{\circ}$ (see Section III). The substrate, which was considered infinite in the simulations, was cut round-shaped with a radius approaching $r_{\mathrm{ext}}$ in order to minimize tag dimensions [Fig. 5(b)].

The measured tag radiation pattern in the $E$-plane and $H$ plane, depicted at Fig. 4(a), exhibits very good agreement with both theory and simulation. The experimental read range, depicted in Fig. 6(b), reaches $R R_{\mathrm{x}}=9.3 \mathrm{~m}$ at $911 \mathrm{MHz}$, which is very close to the simulated value $(9.2 \mathrm{~m}$, Section III). On the other hand, the measured axial read range reaches $R R_{z}=4.2 \mathrm{~m}$, which is higher than the simulated value $(3.4 \mathrm{~m})$, suggesting a value of $X P O L_{\max }=-6 \mathrm{~dB}$, i.e. nearly $3 \mathrm{~dB}$ higher than the simulated value and $4 \mathrm{~dB}$ higher than the theoretical value predicted by means of (12). The authors attribute such a difference to non-idealities in the measurement process, e.g., the non-perfect TEM propagation inside the cell. The tag half- 


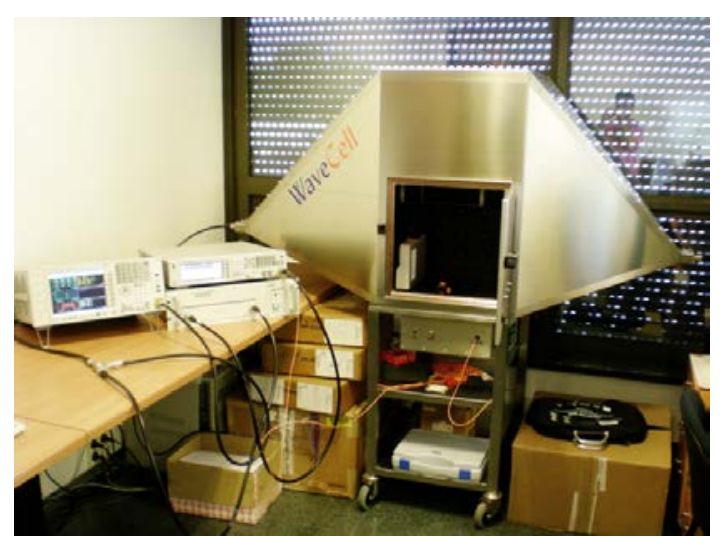

(a)

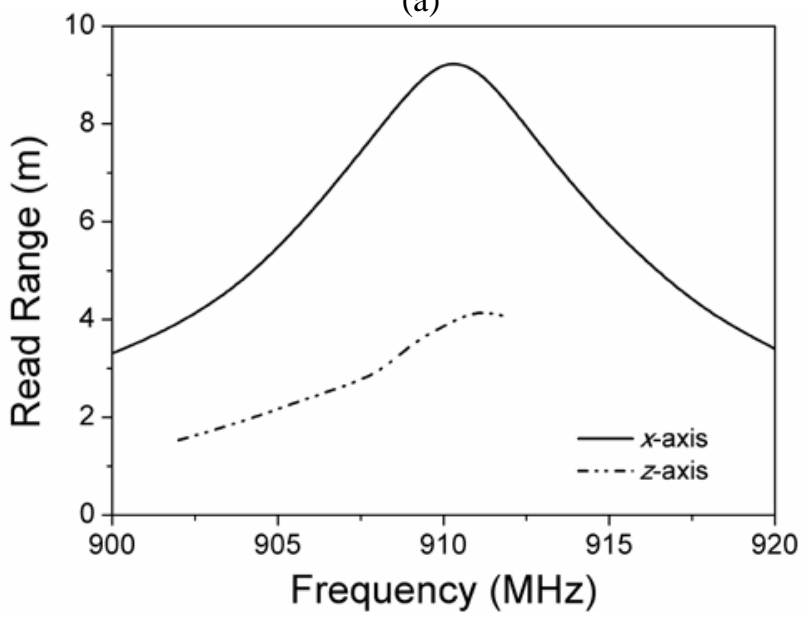

(b)

Fig. 6. (a) Read range measurement setup and (b) tag experimental read range.

power bandwidth, which can be deduced from the read range curve, is $B W=7 \mathrm{MHz}$, which is in good agreement with the simulated bandwidth (8 MHz, Section III). It is interesting to compare the measured value to the value predicted in (22), taking into account the size of the scaled tag and considering $\eta_{\text {rad }}=0.67$ (i.e. the simulated value). The result for the halfpower bandwidth as predicted by (22) is $B W=8 \mathrm{MHz}$, which is in perfect agreement with the simulated value, and very near to the measured one.

As confirmed by measurements, the proposed tag shows good performance in terms of read range, relatively to its dimensions. For comparison, radiation efficiency in the order of $30 \%$ has been reported for a typical meandered antenna of the same electrical size [24]. Moreover, optimization of meander line antennas by means of genetic algorithms suggested an upper bound of roughly $50 \%$ for the radiation efficiency when the antenna maximum size is $\lambda_{0} / 8$ (which is larger than the antenna proposed in this work) [25]. In that case, however, dielectric losses were no taken into account, and the impedance matching network was not included.

It is worth mentioning that, by using the latest ASICs available on the market (e.g., Alien Higgs 4, with $P_{\text {th }}=-18.5 \mathrm{dBm}$, [26], the read range of the proposed tag could be extended up to $13.7 \mathrm{~m}$. Nevertheless, like any other electrically small UHF-RFID tag, performance degradation in terms of radiation efficiency and impedance matching may occur when the tag is attached to some objects, depending on the electromagnetic properties of the labeled item. In many cases this can be taken into account in the tag design stage (e.g., when the object is known a priori) in order to mitigate these effects.

\section{V.CONCLUSION}

The radiation properties of the EC-SRR antenna, in terms of radiation resistance, efficiency, polarization and bandwidth have been studied analytically in this work for the first time. The study has revealed that the structure is suitable to be used as the antenna of a passive UHF-RFID tag, featuring lowprofile, small size, long read range and mitigation of blind spots. The analysis, which has been validated by experimental data (i.e. input impedance and radiation pattern), also has suggested that it is possible to obtain good impedance matching with the RFID ASIC (and with virtually any real impedance, e.g., $50 \Omega$ ) without the need of an impedance matching network. Based on these results, the tag design process has been presented, and a tag prototype has been manufactured. The tag size is $30 \mathrm{~mm} \times 30 \mathrm{~mm}\left(\lambda_{0} / 11 \times \lambda_{0} / 11\right)$, and the measured read range reaches $9.3 \mathrm{~m}$ at $911 \mathrm{MHz}$ in the direction of maximum read range, and $4.2 \mathrm{~m}$ at the same frequency in the direction of minimum read range.

\section{REFERENCES}

[1] N.C. Karmakar, Handbook of Smart Antennas for RFID systems, John Wiley and Sons, NJ, 2010.

[2] R. Das and P. Harrop, Printed and Chipless RFID Forecasts, Players and Opportunities 2009-2019, IdTechEx report, 2009.

[3] GS1 EPCglobal, "Regulatory status for using RFID in the EPC Gen 2 band (860 to $960 \mathrm{MHz}$ ) of the UHF spectrum", 31 Oct 2014.

[4] K. V. S. Rao, P. V. Nikitin, S. F. Lam, "Antenna design for UHF RFID tags: a review and a practical application," IEEE Trans. Ant. Propag., vol. 53, (12), pp. 3870-3876, Dec. 2005.

[5] G. Marrocco, "The art of UHF RFID antenna design: impedancematching and size-reduction techniques," IEEE Ant. Propag. Mag., vol. 50 (1), pp. 66-79, Feb. 2008.

[6] B. D. Braaten, R. P. Scheeler, M. Reich, R. M. Nelson, C. Bauer-Reich, J. Glower and G.J. Owen, "Compact metamaterial-based UHF RFID antennas: deformed omega and split-ring resonator structures," ACES Special Issue on Comput. Experimental Tech. RFID Syst. Applicat., vol. 25, no. 6, 2010

[7] J. B. Pendry, A. J. Holden, D. J. Robbins, W. J. Stewart, "Magnetism from conductors and enhanced nonlinear phenomena," IEEE Trans. Microw. Theory Tech., vol. 47, no. 11, pp. 2075-2084, Nov. 1999.

[8] S.H. Lim, Y.C. Oh, H. Lim, Y.S. Lee, N.H. Myung, "Analysis and design of a UHF RFID tag antenna with a split ring resonator," IEEE International Workshop on Antenna Technology (iWAT), Chiba, pp. 446-449, March 2008.

[9] B.D. Braaten and R.P. Scheeler, "Design of passive UHF RFID tag

[10] antennas using metamaterial-based structures and techniques," INTECH Open Access Publisher, 2010.

[11] R. Marques, F. Martin, M. Sorolla, Metamaterials with Negative Parameters: Theory, Design and Microwave Applications, John Wiley and Sons, New York, 2008.

[12] S.J. Orfanidis, Electromagnetic Waves and Antennas, Rutgers University, 2000.

[13] J. D. Jackson, Classical Electrodynamics (3rd ed.), John Wiley, 1999.

[14] R. Marques, F. Mesa, J. Martel, and F. Medina, "Comparative analysis of edge and broadside coupled split ring resonators for metamaterial design. Theory and experiment," IEEE Trans. Ant. Propag., vol. 51, pp. 2572-2581, Oct. 2003. 
[15] C. A. Balanis, Antenna Theory: Analysis and Design, 3rd Ed., WileyInterscience, 2005

[16] M. Polivka, A. Holub, M. Vyhnalik, M. Svanda, "Impedance properties and radiation efficiency of electrically small double and triple split-ring antennas for UHF RFID applications,” IEEE Ant. Wireless Propag. Lett., vol. 12, pp. 221-224, March 2013.

[17] M. Gustafsson, C. Sohl, G. Kristensson, "Illustrations of new physical bounds on linearly polarized antennas," IEEE Trans. Ant. Propag., vol. 57(5), pp. 1319-1327, May 2009.

[18] H.A. Wheeler, "Fundamental limitations of small antennas," Proc. IRE, vol. 69, pp. 1479-1484, Dec. 1947.

[19] L.J. Chu, "Physical limitations of omni-directional antennas," J. Appl. Phys., vol. 19, no. 12, pp. 1163-1175, Dec. 1948.

[20] K. Mohammadpour-Aghdam, R. Faraji-Dana, G.A.E. Vandenbosch, S. Radiom and G.G.E. Gielen, "Physical bound on Q factor for planar antennas," Proc. 41st European Microwave Conf., pp. 250-252, Oct. 2011.

[21] J.S. McLean, "A re-examination of the fundamental limits on the radiation Q of electrically small antennas," IEEE Trans. Ant. Propag., vol. 44(5), pp. 672-676, May 1996.

[22] A.D. Yaghjian, S.R. Best, "Impedance, bandwidth, and Q of antennas," IEEE Trans. Ant. Propag., vol. 53, no. 4, pp. 1298-1324, Apr. 2005.

[23] G. Zamora, F. Paredes, F.J. Herraiz-Martínez, F. Martín and J. Bonache, "Bandwidth limitations of ultra high frequency-radio frequency identification tags," IET Microwaves Antennas and Propagation, vol. 7, no. 10, pp. 788-794, July 2014.

[24] X. Qing, C. K. Goh. and Z. N. Chen, "Impedance characterization of RFID tag antennas and application in tag co-design,” IEEE Trans. Microw. Theory Tech, vol 57, no. 5, pp. 1268-1274, 2009.

[25] M. Takiguchi and Y. Yamada, "Input impedance increase of a very small meander line antenna," IEEE Antennas and Propagation Society International Symposium, pp. 856-859, 2003.

[26] G. Marrocco, "Gain-optimized self-resonant meander line antennas for RFID applications," IEEE Ant. Wireless Propag. Lett., vol. 2, pp. 302305, 2003.

[27] Alien Higgs $^{\mathrm{TM}}-4$ datasheet, Available online at www.alientechnology.com

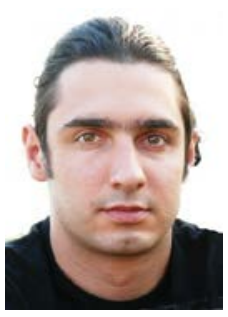

Simone Zuffanelli was born in Prato (Italy) in 1983. He received the Electronics Engineering Diploma in 2008 at the Università Degli Studi di Firenze. He obtained "Micro and Nanoelectronics Engineering" master's degree in 2011 at the Universitat Autònoma de Barcelona. He is currently working as a researcher in the field of metamaterial inspired antennas and RFID tags. His previous experiences include electronic design in the context of European projects "Persona" and "NOMS".

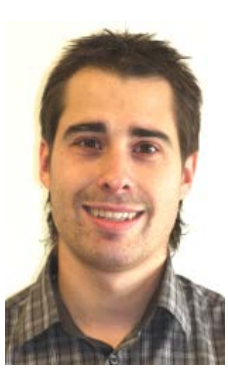

Gerard Zamora was born in 1984 in Barcelona (Spain). He received the Telecomunications Engineering Diploma, specialising in Electronics from the Universitat Autònoma de Barcelona in 2005. He obtained the Telecomunications Engineering degree in 2008 and the $\mathrm{PhD}$ degree from the same university in 2013. He is currently working as a researcher at the Universitat Autònoma de Barcelona. His research interests include antennas and microwave devices, the RFID technology and metamaterials.

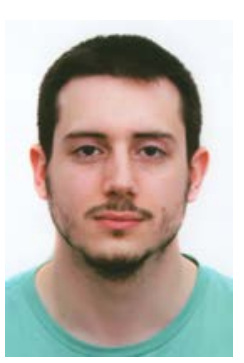

Pau Aguilà was born in Barcelona, Spain in 1989. He received the Telecommunications Engineering diploma (specializing in electronics) and the Telecommunications Engineering degree from the Universitat Autònoma de Barcelona in 2010 and 2012, respectively. $\mathrm{He}$ is currently working toward his $\mathrm{PhD}$ degree at the Universitat Autònoma de Barcelona in the field of metamaterials applied to microwave devices and RFID systems.

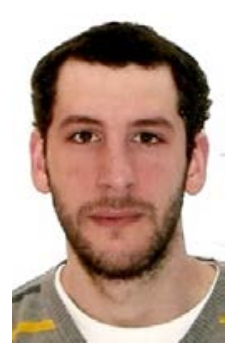

Ferran Paredes was born in Badalona (Barcelona), Spain in 1983. He received the Telecommunications Engineering Diploma (specializing in Electronics) and the Telecommunications Engineering degree from the Universitat Autònoma de Barcelona in 2004 and 2006, respectively and the $\mathrm{PhD}$ degree in Electronics Engineering from the same university in 2012. He was Assistant Professor from 2006 to 2008 at the Universitat Autònoma de Barcelona, where he is currently working as a Research Assistant. His research interests include metamaterial concepts, passive microwaves devices,

antennas and RFID.

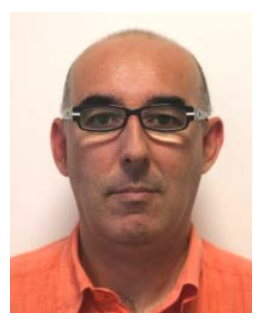

Ferran Martín (M’04-SM'08-F'12) was born in Barakaldo (Vizcaya), Spain in 1965. He received the B.S. Degree in Physics from the Universitat Autònoma de Barcelona (UAB) in 1988 and the $\mathrm{PhD}$ degree in 1992. From 1994 up to 2006 he was Associate Professor in Electronics at the Departament d'Enginyeria Electrònica (Universitat Autònoma de Barcelona), and since 2007 he is Full Professor of Electronics. In recent years, he has been involved in different research activities including modelling and simulation of electron devices for high frequency applications, millimeter wave and $\mathrm{THz}$ generation systems, and the application of electromagnetic bandgaps to microwave and millimeter wave circuits. He is now very active in the field of metamaterials and their application to the miniaturization and optimization of microwave circuits and antennas. He is the head of the Microwave Engineering, Metamaterials and Antennas Group (GEMMA Group) at UAB, and director of CIMITEC, a research Center on Metamaterials supported by TECNIO (Generalitat de Catalunya). He has organized several international events related to metamaterials, including Workshops at the IEEE International Microwave Symposium (years 2005 and 2007) and European Microwave Conference (2009), and the Fifth International Congress on Advanced Electromagnetic Materials in Microwaves and Optics (Metamaterials 2011), where he has acted as chair of 
the Local Organizing Committee. He has acted as Guest Editor for three Special Issues on Metamaterials in three International Journals. He has authored and co-authored over 450 technical conference, letter, journal papers and book chapters, he is co-author of the book on Metamaterials entitled Metamaterials with Negative Parameters: Theory, Design and Microwave Applications (John Wiley \& Sons Inc. 2008), and he has generated 15 PhDs. Ferran Martín has filed several patents on metamaterials and has headed several Development Contracts.

Prof. Martín is a member of the IEEE Microwave Theory and Techniques Society (IEEE MTT-S). He is reviewer of the IEEE Transactions on Microwave Theory and Techniques and IEEE Microwave and Wireless Components Letters, among many other journals, and he serves as member of the Editorial Board of IET Microwaves, Antennas and Propagation and International Journal of RF and Microwave Computer-Aided Engineering. He is also a member of the Technical Committees of the European Microwave Conference (EuMC) and International Congress on Advanced Electromagnetic Materials in Microwaves and Optics (Metamaterials). Among his distinctions, Ferran Martín has received the 2006 Duran Farell Prize for Technological Research, he holds the Parc de Recerca UAB Santander Technology Transfer Chair, and he has been the recipient of two ICREA ACADEMIA Awards (calls 2008 and 2013).

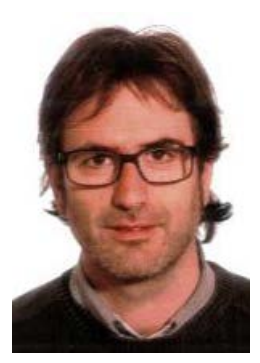

Jordi Bonache (S'05-M'07) was born in 1976 in Barcelona, Spain. He received the Physics and Electronics Engineering degrees and the Ph.D. degree in electronics engineering from the Universitat Autònoma de Barcelona, Barcelona, Spain, in 1999, 2001, and 2007, respectively. In 2000, he joined the "High Energy Physics Institute" of Barcelona (IFAE), where he was involved in the design and implementation of the control and monitoring system of the MAGIC telescope. In 2001, he joined the Department of Electronics Engineering of the Universitat Autònoma de Barcelona where he is currently Lecturer. From 2006 to 2009 he worked as executive manager of CIMITEC. Currently he is leading the research in RFID and antennas in CIMITEC. His research interests include active and passive microwave devices, metamaterials, antennas and RFID.

Post-print of: Zuffanelli, et al. "Analysis of the split ring resonator (SRR) antenna applied to passive UHF-RFID tag design" in IEEE transactions on antennas and propagation, vol. 64, issue 3 (March 2016), p. 856-864. DOI 10.1109/ TAP.2015.2513084 - Cop. 2016 IEEE. Personal use of this material is permitted. Permissions from IEEE must be obtained for all other uses, in any current or future media, including reprinting/republishing this material for advertising or promotional purposes, creating new collective works, for resale or redistribution to servers or lists, or reuse of any copyrighted component of this work in other works. 